\title{
Modeling and Analyzing the Impact of Location Inconsistencies on Geographic Routing in Wireless Networks *
}

\author{
Yongjin Kim ${ }^{a}$ \\ Jae-Joon Lee ${ }^{a}$ \\ Ahmed Helmy ${ }^{a}$ \\ yongjkim@usc.edu \\ jaejlee@usc.edu \\ helmy@usc.edu \\ ${ }^{a}$ EE Department, University of Southern California, Los Angeles, CA, U.S.A
}

Recently, geographic routing in wireless networks has gained attention due to several advantages of location information. Location information eliminates the necessity to set up and maintain explicit routes, which reduces communication overhead and routing table size. These advantages allow scalability especially in dynamic and unstable wireless networks. However, no matter which technologies or techniques a location system uses, its measurements will have some amount of quantifiable inaccuracy depending on environment and system. These inaccuracies may affect the performance and even correctness of geographic routing. However, thus far, these impacts have not been studied in-depth. In this paper, we analyze the impact of location inaccuracy on geographic routing. First, we model location inaccuracy metrics - absolute location inaccuracy, relative distance inaccuracy, absolute location inconsistency and relative distance inconsistency. Then, we analyze how location inaccuracy metrics affect the building blocks of geographic routing including greedy forwarding and local maximum resolution. We use extensive NS-2 simulations to evaluate the performance of geographic routing using a wide array of parameter settings including inaccuracy level, node degree and network diameter. In our simulation results, reasonable location inaccuracy (of 20 percent or less of radio range) caused packet drop reaching up to 54 percent, non-optimal path up to 53 percent and packet looping. These observations indicate the importance of re-visiting geographic routing protocols, and the significance of considering location inaccuracy in their design and evaluation.

\section{Introduction}

In recent years, wireless communication devices are gaining tremendous popularity due to the advance of hardware technology and various commercial applications such as conferencing, home networking, emergency services, embedded computing, and sensor dust, among others. This stimulated the introduction of ad hoc networks that do not have a preestablished network infrastructure. In such ad hoc networks, especially in mobile ad hoc networks where nodes may move arbitrarily, routing is a challenging task because of frequent topology change without prior notice. Many routing protocols for ad hoc networks have been proposed and existing ad hoc routing protocols can be divided into two approaches: topology-based[4] and position-based routing[12]. Topology-based routing, such as table-driven or on-demand routing, uses link information of the networks to perform packet forwarding. Position-based routing uses physical location information to perform packet forwarding.

*Ahmed Helmy was supported by Grants from NSF CAREER, Intel and Pratt\&Whitney ICT
Geogrphic routing $[1,6,10,12,20,21]$ has been given attention recently due to its several merits against traditional topology-based routing. That is, geographic routing eliminates some of the limitations of topology-based routing by using location information. In geographic routing, the routing decision at each node is based on the destination's location contained in the packet header and the location of the forwarding node's neighbors. Geographic routing thus does not require the establishment or maintenance of routes. The nodes have neither to store routing tables nor to transmit messages to keep routing tables up to date. Hence, geographic routing allows routers to be nearly stateless, and requires propagation of topology information for only a single hop. This nature of location is the key to location's usefulness providing following merits.

- Lower communication overhead to set up and maintain routes

- Small routing table

- Natural extention to geocasting using location information 
Location information has important meaning especially in sensor networks. In sensor networks, location information may be used for various purposes, such as event reporting at specific location, data centric storage[18], or naming schemes[5]. Accurate location information is considered important especially in routing and naming schemes. Because of dense nature of sensor networks, small location inaccuracy can affect the performance and correctness of sensor networks.

Because of the many merits of location information in wireless networks mentioned above, geographic routing protocol is under active research. However, no matter which technology is used, location information has some inaccuracy depending on the localization system and the environment. Nodes could acquire the estimates of their locations from outside sources such as GPS[15,19]. GPS has inheretent inaccuracy. In addition, it is not justifiable from economic and energy preservation points of view to equip each node with a GPS receiver in the entire wireless networks. The alternative solution proposed is to design a location discovery algorithm that uses measurements of the distances between nodes and estimates of the locations of a small percentage of nodes( acquired through GPS, for example ) to determine the locations of all or majority of nodes in a network. Distance measurement is also inherently noisy. Mobility during beacon interval may also induce location inaccuacy. Transient state of location information may induce inconsistent view of location information, which may result in serious problems in geographic routing.

Furthermore, even if every node knows its own location, there is still a need for an underlying location discovery and dissemination service. Location errors and inconsistencies may also be introduced by location dissemination services, such as the grid location service (GLS) [6].

Existing geographic routing protocols assume accurate location information, which is impractical in real world. As a result, there has been little analysis how location inaccuracy affects geographic routing protocol. It is essential to know the impact of location inaccuracy on geographic routing. In this paper, we investigate the impact of location inaccuracy on geographic routing. We first model location inaccuracy metrics: absolute location inaccuracy, relative distance inaccuracy, absolute location inconsistency and relative distance inconsistency. Each inaccuracy metric may affect geogrphic routing in terms of performance - such as packet drop, non-optimal path and routing loop and even correctness of protocol. Then we study how location inaccuracy metrics affect the building blocks of geographic routing including greedy forwarding and local maximum resolution using simulation and analysis. Different node degree and network diameter with varying inaccuracy level have different impact on geographic routing. In our simulation, location inaccuracy caused packet drop rate up to 54 percent and non-optimal path up to 53 percent in dense network. Routing loop occurred (up to 1.9 percent) in sparse network. We used local maximum resolution scheme of GPSR[1] as a case study and found that location inaccuracy affects even correctness of location maximum resolution using analysis and simulation.

It is interesting to note that we observed some benefits for location inaccuracy in some scenarios of greedy forwarding, but those benefits were overshadowed by significant performance degradation.

In all, our observations strongly suggest that the design of geographic routing protocols should be revisited and that it is essential to consider location inaccuracy and inconsistency in the design and evaluation of geographic routing.

The rest of paper is organized as follows. In section II, we briefly review geographic routing. Section III defines four inaccuracy metrics - absolute location inaccuracy, relative distance inaccuracy, absolute location inconsistency and relative distance inconsistency - and discusses possible problems of the inaccuracy metrics. Section IV investigates whether location inaccuracy always has a bad impact on geographic routing. Section V shows the impact of location inaccuracy on greedy packet forwarding. In section VI, we show how location inaccuracy affects local maximum resolution using perimeter mode of GPSR as a case study. Section VII concludes our paper.

\section{Background and Related Work}

Geographic routing can be categorized into three approaches depending on the forwarding strategies[12]: Greedy packet forwarding, restricted directional flooding and hierarchical routing.

Using greedy packet forwarding, a sender of a packet includes the position of the destination in the packet header. This information is gathered by appropriate location service system. A sender sends a packet to the closest node to the destination within transmission range as shown in figure 1.

Ideally, this process can be repeated through intermediate nodes until the destination has been reached. One function of greedy forwarding is to compare distance between all neighboring nodes - within transmission range - and the destination node and decides 


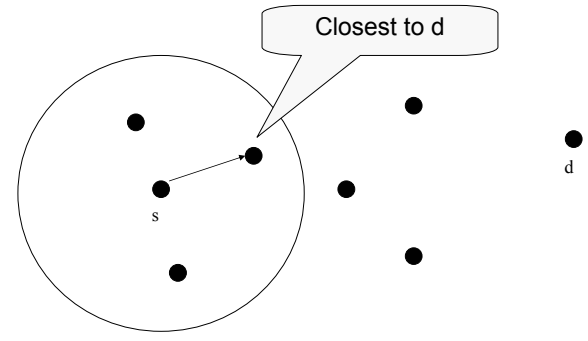

Figure 1: Greedy Packet Forwarding

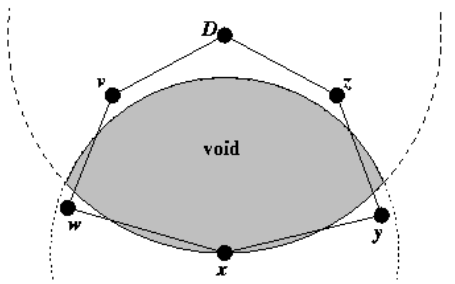

Figure 2: Local Maximum

the closest neighbor to the destination. The other function of greedy forwarding is to decide whether it is in local maximum situation or not, where there exists no neighbor node closer to the destination than the forwarding node. The proper operation of these two functions can be affected by location inaccuracy. That is, location inaccuracy increases the probability of making incorrect neighbor selection and wrong greedy decision and consequent packet drop, nonoptimal path and packet looping. We define wrong greedy decision as selecting a node which is not the closest to the destination within transmission range. We will verify those phenomena in simulation. Even though greedy forwarding can find optimal path to the destination, there are some situations in which greedy forwarding fails, which is called local maximum. Local maximum occurs when greedy forwarding faces a dead end and fails to find a path between a sender and a destination, even though one does exist. A local maximum scenario is illustrated in figure 2 .

As seen in the figure, a local maximum scenario oc- curs when there are no nodes in the void region. This situation happens because of the greedy nature. Currently, the most advanced scheme to address this local maximum is graph-based scheme such as face-2 [14] and perimeter mode [1] with planar graph. To prevent loops, constructing a plannar graph that does not have a cross edge among the nodes is the most important function. However, as we shall show, constructing a plannar graph poses great challenges in the presence of location inaccuracy. We will study how absolute location inaccuracy affects plannar graph construction using analysis and simulation.

Restricted directional flooding approach is developed to relieve high overhead problem of global flooding in geographic routing. LAR[20] is one example. LAR proposes the use of position information to enhance the route discovery phase of reactive ad hoc routing approaches. Reactive ad hoc routing protocols frequently use flooding as a means of route discovery. Under the assumption that nodes have information about other nodes' location, this location information can be used by LAR to restrict the flooding to a certain area. LAR defines an expected zone of the destination based on available location information. That is, the expected zone is the place where the destination may be located during a certain time interval. The expected zone can be calculated depending on initial location, time interval and mobility speed. LAR also defines a request zone as the set of nodes that should forward the routing discovery packet. The request zone typically includes the expected zone. Node will forward the route discovery packet only if they are within that specific region.

In traditional networks, the complexity each node has to handle can be reduced tremendously by establishing some form of hierarchy. Hierarchical routing allows those networks to scale to a very large number of nodes. One approach that combines hierarchical and geographic routing is part of the Terminodes project[10]. In Terminodes routing, a two-level hierarchy is proposed. Packets are routed according to a proactive distance vector scheme if the destination is close to the sending node. For long distance routing a greedy approach is used. Once a packet reaches the area close to the recipient, it continues to be forwarded by means of the local routing protocol. It is shown that the introduction of a hierarchy can significantly improve the ratio of successfully delivered packets and the routing overhead compared to reactive ad hoc routing algorithms.

In this paper, we focus on the greedy packet forwarding scheme which is considered a basic mecha- 
nistic building block in geographic routing. In addition, we analyze how location inaccuracy affects the local maximum resolution mechanism - a mechanism sufficient to provide correct geographic routing in the absence of location inaccuracy. To our knowledge, this is one of the first in-depth studies of the effects of location inconsistencies on geographic routing protocols. Preliminary work in [7][8][9] provides algorithmic analysis of face routing in presence of location inaccuracy (vs. inconsistency) in static sensor networks, and reaches similar conclusions that the effects are significant. Preliminary work in [3] investigates effects of mobility on geographic routing. Our work is unique in providing the analysis for location inconsistencies, mainly in ad hoc networks. There is a large body of work on localization in wireless networks. We only cite a limited number of studies for illustration. A more detailed treatment of this topic is beyond the scope of this paper. GPS[15] is a common technique for localization. GPS introduces localization errors that depend on the receiver used (among other factors). Those errors range from $1 \mathrm{~cm}-100 \mathrm{~m}$ (with $10 \mathrm{~m}-100 \mathrm{~m}$ mean error incurred for reasonable cost receivers). GPS may not work in-doors, and it may not be feasible or economic for classes of ad hoc networks (including sensor networks) to have GPS capability. GPS-less techniques have been recently designed for wireless networks. Reference beacons are used for localization in[13], where the accuracy increases with increased number of reference beacons and is a function of the radio range. The study cites 20 percent or more (of the radio range) as the mean localization error with high beacon deployment. We borrow from these studies to develop our location error model. All studies of localization include a section on localization error, and all studies cite non-negligible localization errors.

\section{Inconsistency Metrics}

In this section, we classify location inconsistency into four metrics - Absoulte location inaccuracy, relative distance inaccuracy, absolute location inconsistency and relative distance inconsistency. Each of these metrics potentially has a negative impact on geographic routing in terms of packet drop, non-optimal path and routing loop.

We do not assume any specific location system such as [6,16,22]. Instead, we focus on the general location inconsistency that is incurred in any location system depending on its implementation and the deployment environment.

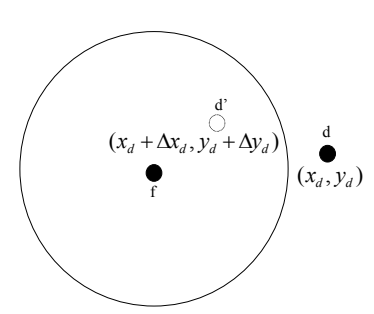

Figure 3: Packet Drop by Absolute Location Inaccuracy

\section{III.A. Absolute Location Inaccuracy}

We define the absolute location inaccuracy for each node $i$ as follows.

$$
\sqrt{\left(x_{i}-\left(x_{i}+\triangle x_{i}\right)\right)^{2}+\left(y_{i}-\left(y_{i}+\triangle y_{i}\right)\right)^{2}}
$$

where, $A_{i}\left(x_{i}, y_{i}\right)$ is the true location and $A_{i}\left(x_{i}+\right.$ $\left.\triangle x_{i}, y_{i}+\triangle y_{i}\right)$ is the faulty location. Absolute location inaccuracy is the distance between the true location and the faulty location. A possible problem of absolute location inaccuracy is packet drop. As illustrated in figure 3 , node $i$ perceives the destination node $d$ is at $\left(x_{d}+\triangle x_{i}, y_{i}+\triangle y_{i}\right)$. However, the true location of node $d$ is $\left(x_{d}, y_{d}\right)$ which is outside the transmission range. Absolute location inaccuracy may induce relative distance inaccuracy, absolute location inconsistency and relative location inconsistency. We define each of terms in the following sections.

\section{III.B. Relative Distance Inaccuracy}

In figure 4 , the distance between node $i$, node $j$ and the destination node $d$ can be calculated as follows.

$$
\begin{aligned}
& \text { Dist }_{i}=\sqrt{\left(x_{d}-x_{i}\right)^{2}+\left(y_{d}-y_{i}\right)^{2}} \\
& \text { Dist }_{j}=\sqrt{\left(x_{d}-x_{j}\right)^{2}+\left(y_{d}-y_{j}\right)^{2}}
\end{aligned}
$$

Then the true relative distance is defined as follows.

$$
\triangle \text { Dist }=\text { Dist }_{i}-\text { Dist }_{j}
$$

Greedy forwarding algorithm selects node $i$ if

$$
\triangle \text { Dist }<0
$$




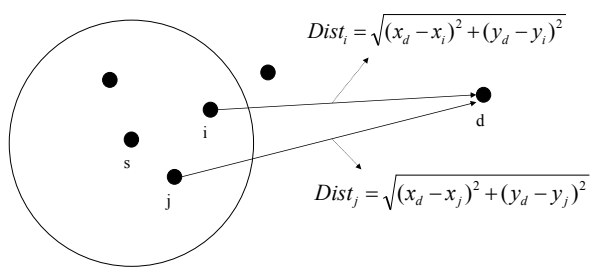

Figure 4: Distance between Node and Destination

which means $i$ is closer to the destination than $j$. However, greedy forwarding may fail if high relative distance inaccuracy exists. That is, a forwarding node may select a node which is not the closest to the destination within its radio range. Before we define relative distance inaccuracy, we define distance inaccuracy between node $i$ and the destination $d$ and between node $j$ and the destination $d$ as follows.

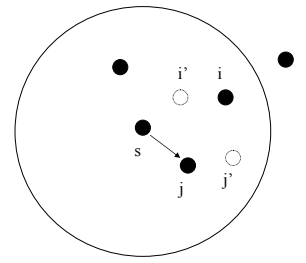

Figure 5: Wrong Greedy Decision. The black circle represents the true location of a node and the white circle represents the faulty location.

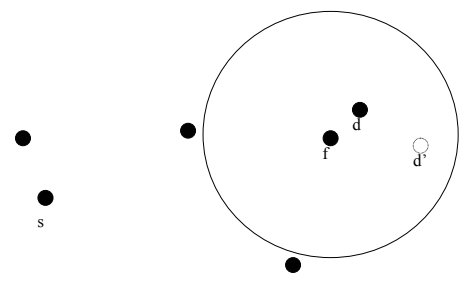

$\begin{array}{cl}\text { Dist }_{j-\text { error }}= & \begin{array}{l}\text { Figure 6: False Local Maximum by Absolute Loca- } \\ \text { tion Inconsistency }\end{array} \\ \sqrt{\left(\left(x_{d}+\triangle x_{d}\right)-\left(x_{j}+\triangle x_{j}\right)\right)^{2}+\left(\left(y_{d}+\triangle y_{d}\right)-\left(y_{j}+\triangle y_{j}\right)\right)^{2}}\end{array}$

Then, the relative distance inaccuracy is defined as follows.

$$
\triangle \text { Dist } \cdot \triangle \text { Dist }_{\text {error }} \leq 0
$$

where,

$$
\triangle \text { Dist }_{\text {error }}=\text { Dist }_{i-\text { error }}-\text { Dist }_{j-e r r o r}
$$

Possible problems of relative distance inaccuracy include higher packet drop rate due to false local maximum and non-optimal routing path due to wrong greedy decision. We define false local maximum as the situation where forwarding node could not find the closer neighbor node to the destination due to location inaccuracy even though there is in fact closer neighbor node to the destination. This is shown in figure 5 , where the forwarding node $s$ selects node $j$ even though node $i$ is the closest node to the destination within the transmission range. We shall validate and quantify the impact of those possible problems in the simulation section.

\section{III.C. Absolute Location Inconsistency}

We define absolute location inconsistency as follows.

Absolute Location Inconsistency $=$

$$
\sqrt{\left(x_{k}^{i}-x_{k}^{j}\right)^{2}+\left(y_{k}^{i}-y_{k}^{j}\right)^{2}}
$$

where $\left(x_{k}^{i}, y_{k}^{i}\right)$ is the location of node $k$ as perceived by node $i$ and $\left(x_{k}^{j}, y_{k}^{j}\right)$ is the location of node $k$ as perceived by node $j$.

Absolute location inconsistency represents the difference of the same target locations perceived by two nodes. One potential problem of high absolute location inconsistency is the false local maximum within the range reachable to the destination. An example of absolute location inconsistency is illustrated in figure 6. Sender node $s$ includes the destination location in packet header as location $d$. However, a forwarding node, $f$, at the last hop perceives the destination at a different location $d^{\prime}$. If distance 
between the $d^{\prime}$ and $d$ is greater than the distance between $f$ and $d$, false local maximum occurs.

If global addressing is not available (or not used, as in some sensor networks) and the geographic routing algorithm relies only on the location information, there is a high probability that the packet cannot be delivered to the destination when absolute location inconsistency is high with high node density in radio range since the forwarding node cannot find a neighbor node closer to the destination than itself. This is especially the case when location is used as a naming in sensor networks [5]. We call this situation as the false local maximum within the destination range.

Absolute location inconsistency may induce relative distance inconsistency, which is explained next.

\section{III.D. Relative Distance Inconsistency}

We define distance inaccuracy perceived by node $i$ as follows.

$$
\triangle D i s t_{\text {error }}^{i}=D i s t_{i-\text { error }}^{i}-D i s t_{j-e r r o r}^{i}
$$

In addition, we define distance inaccuracy perceived by node $j$ as follows.

$$
\triangle D i s t_{\text {error }}^{j}=D i s t_{i-\text { error }}^{j}-D i s t_{j-e r r o r}^{j}
$$

Then relative distance inconsistency is be defined as follows.

$$
\triangle D i s t_{\text {error }}^{i} \cdot \triangle D i s t_{\text {error }}^{j} \leq 0
$$

One potential problem of relative distance inconsistency is formation of routing loop. A routing loop case is illustrated in figure 7 . Node $s$ thinks that node $j$ is the closest node to the destination within the transmission range and sends the packet to $j$. In turn, node $j$ thinks that node $i$ is the closest node to the destination and send a packet back to the node $i$ and this continuation results in routing loop. This situation can occur at the sender's transmission range or in the middle of path.

There can be multi-hop relative distance inconsistency even though there is no relative distance inconsistency between two direct neighbor. Figure 8 illustrates multi-hop routing loop. Sender node $s$ sends a packet to node $j$. Then, node $j$ sends a packet to the node $i$ and node $i$ sends a packet to the node $k$. Node $k$ sends the packet back to node $j$ and so on , forming a multi-hop loop. This situation frequently occurred in our simulations.

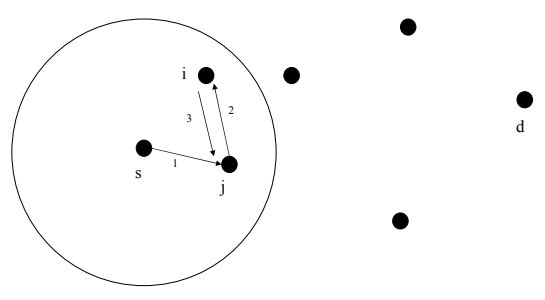

Figure 7: Routing Loop caused by Relative Distance Inconsistency

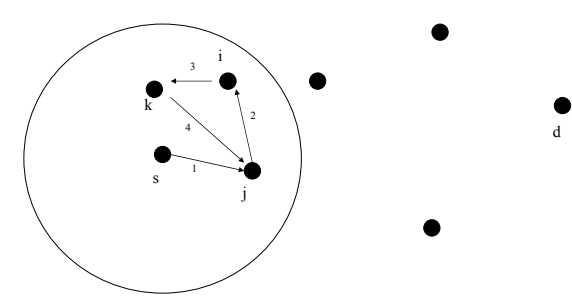

Figure 8: Multi-hop Routing Loop caused by Relative Distance Inconsistency

We summarize the four inaccuracy metrics in table I.

\section{Is Location Inconsistency Always Bad?}

The answer is 'not always'. For example, packet drop in greedy forwarding due to a local maximum scenario (with precise location) may be avoided if location inaccuracy leads to a valid greedy forwarding path. Local maximum occurs in following condition.

$$
\{i \mid d(s, d)>d(i, d)\}=\{\}
$$

Where $d(s, d)$ is distance between $s$ and $d$ and $s$ is sending node, $d$ is destination node and $i$ is neighbor node. However, local maximum can be addressed by inaccuracy if,

$$
\{i \mid d(s, d)>d(i, d)\} \neq\{\}
$$

However, the one of the following conditions should be satisfied. 
Table 1: Inconsistency Metrics

\begin{tabular}{|c|c|c|}
\hline \multirow{2}{*}{ Inaccuracy } & Absolute Location Inaccuracy & $\sqrt{\left(x_{i}-\left(x_{i}+\triangle x_{i}\right)\right)^{2}+\left(y_{i}-\left(y_{i}+\triangle y_{i}\right)\right)^{2}}$ \\
\cline { 2 - 3 } & Relative Distance Inaccuracy & $\triangle$ Dist $\cdot \triangle$ Dist error $\leq 0$ \\
\hline \multirow{2}{*}{ Inconsistency } & Absolute Location Inconsistency & $\sqrt{\left(x_{k}^{i}-x_{k}^{j}\right)^{2}+\left(y_{k}^{i}-y_{k}^{j}\right)^{2}}$ \\
\cline { 2 - 3 } & Relative Distance Inconsistency & $\triangle D i s t_{\text {error }}^{i} \cdot \triangle$ Dist $_{\text {error }}^{j} \leq 0$ \\
\hline
\end{tabular}

$$
\{i \mid \text { Path between } i \text { and } d \text { exists }\} \neq\{\}
$$

or,

$$
\{i \mid d(i, d)<\text { Transmission Range }\} \neq\{\}
$$

This provides a stringent condition in the presense of location inaccuracy in order for location inaccuracy to have good impact on geographic routing. We shall re-examine this fact in the simulation section.

\section{Simulation of Greedy Forwrding}

We perform extensive simulations in NS-2[11] to investigate the impact of location inaccuracy on geographic routing. The original NS-2 code for GPSR was used. We use $250 \mathrm{~m}$ of radio range and Gaussian distributions (with zero mean) with different standard deviation to generate location inaccuracy. Location inaccuracy is added to true location. In our simulation, location error is represented as the fraction of standard deviation over radio range since a different radio range may be used in different networks. For example, if the percentage location inaccuracy is 10 percent this means that the standard deviation for the Gaussian distribution is 10 percent of the radio range. Different parameters for node degree and network diameter are used. Node degree is defined as the average number of neighbor nodes. We use different network diameter, 750x750, 1050x1050, 1350x1350, with the same node degree of 17 . We averaged 5 simulations for each node degree, network diameter and location error. Simulation parameters are in table II. We did not add mobility to investigate only the impact of location inaccuracy. Mobility is expected to exacerbate the inaccuracy problem leading to even higher percentage inaccuracy. We perform simulation on greedy mode without local maximum resolution first, then we perform analysis and simulation of perimeter mode of GPSR as a case study of local maximum resolution.

\section{V.A. Impact on Drop Rate}

Figure 9 shows the effect of node degree and location error on packet drop rate. It is clear that as location error gets larger then the drop rate becomes higher. By carefully analyzing the simulation traces, we observe that the main reason for packet drop is false local maximum. A packet is forced to drop if there is no local maximum resolution mechanism under false local maximum situation. Even if there is local maximum resolution, longer detour is inevitable and local maximum resolution itself may also have some problem with location inaccuracy, which will be discussed in the section VI. Figure 10 shows false local maximum rate. We can see that as location inaccuracy gets higher, false local maximum rate significantly increases up to 30 percent. Figure 11 shows that in case of dense network, almost over 90 percent of the packet drop happens within the destination radio range. The reason for higher packet drop in dense network is the higher probability of false local maximum within the destination range due to absolute location inconsistency that was explained earlier in section III-C. Figure 12 shows the effect of network diameter on the packet drop rate. Network diameter is changed from $750 \times 750$ to $1350 \times 1350$ with the same node degree of 17. We generated location inaccuracy with standard deviation of $25 \mathrm{~m}$. Drop rate gets higher as the network gets larger reaching up to 43 percent. This is because of the fact that as the network gets larger, there is higher probability of relative distance inaccuracy and absolute location inconsistency.

\section{V.B. Impact on Optimal Path}

We investigate the impact of location inaccuracy on optimal path rate. Figure 13 shows the non-optimal path rate. There is higher non-optimal path rate in dense network reaching up to 53 percent. The reason can be explained with figure 14, which shows the wrong greedy neighbor decision rate. Since the distance between nodes is closer in denser network, the rate of wrong greedy neighbor decision is higher. This shows the more frequent relative distance inaccuracy 
Table 2: Simulation Parameters

\begin{tabular}{|l|l|}
\hline Parameter & Value \\
\hline \hline Radio Range & $250 \mathrm{~m}$ \\
\hline Standard Deviation of Inaccuracy & $0,10,20,30,40,50 \mathrm{~m}$ \\
\hline Location error & $0,5,10,15,20 \%$ \\
\hline Node Degree & $5,8,19($ area $: 1350 \mathrm{mx} 1350 \mathrm{~m})$ \\
\hline Network Diameter & $750 \mathrm{mx} 750 \mathrm{~m}, 1050 \mathrm{mx} 1050 \mathrm{~m}, 1350 \mathrm{~m} \times 1350 \mathrm{~m}$ \\
\hline Traffic Sources & 30 \\
\hline
\end{tabular}

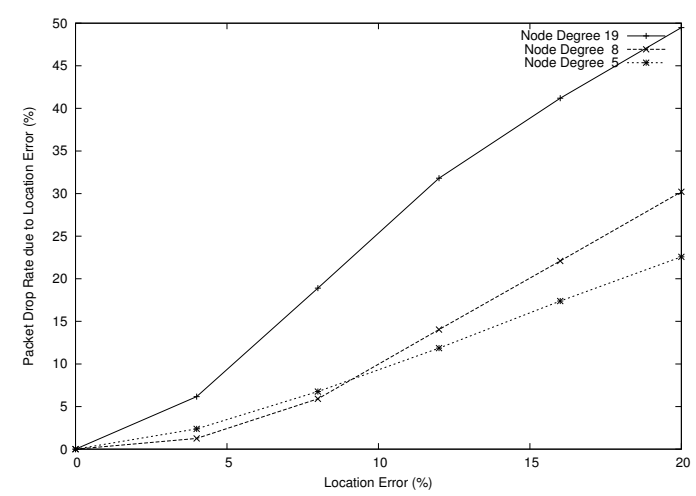

Figure 9: Impact of Location Inaccuracy and Node Degree on Packet Drop Rate

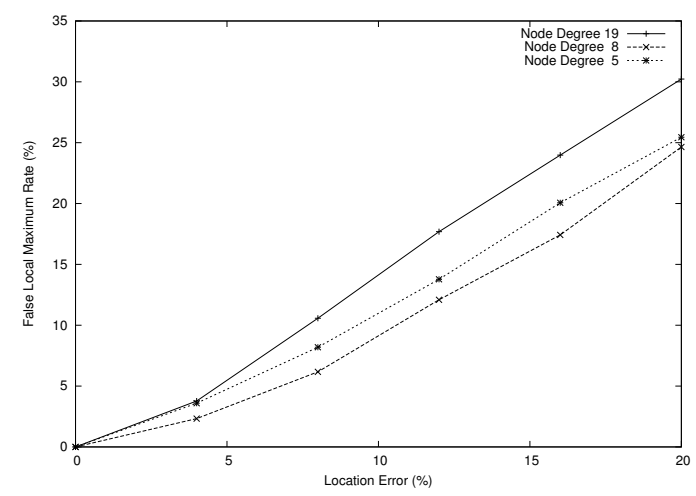

Figure 10: False Local Maximum Rate

in denser network. This results in more non-optimal paths. The number of non-optimal path gets higher as network diameter gets large (Figure 15), due to increased probability of wrong neighbor selection.

\section{V.C. Impact on Looping}

In our simulation routing loops occurred because of relative distance inconsistency. Figure 16 shows that packet drop due to loop. There is more probability of relative distance inconsistency in sparse network, resulting in more routing loops. Single hop loop and multi-hop loop occurred in our simulation. Packets

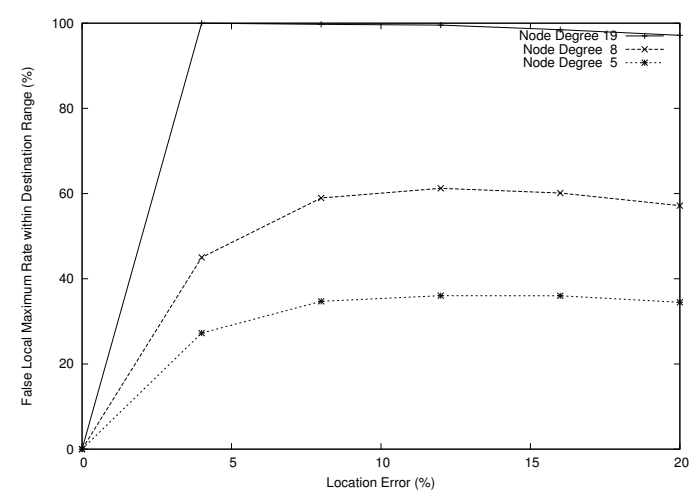

Figure 11: False Local Maximum Rate within the

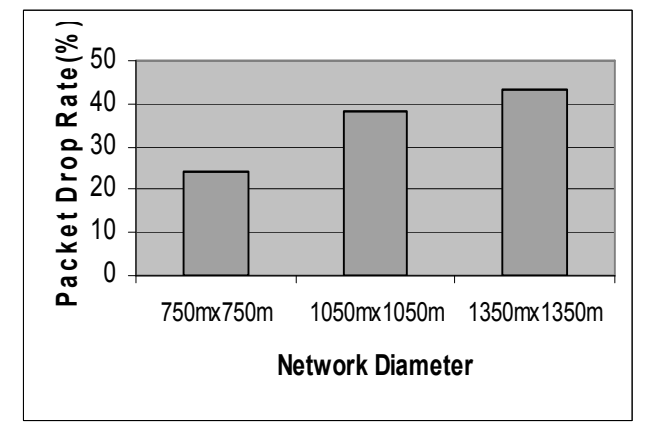

Figure 12: Impact of Network Diameter on Packet Drop Rate

traversing the loop consume node power and cause contention in network path, which significantly consumes the network resources and affects the overall network performance.

\section{V.D. Good Impact vs. Bad Impact}

As we mentioned in section IV, theoretically there can be good effects of location inaccuracy on routing performance in terms of packet drop rate. However, in our extensive simulation, the percentage of good impact was very low (Figure 17). The bad effects far 


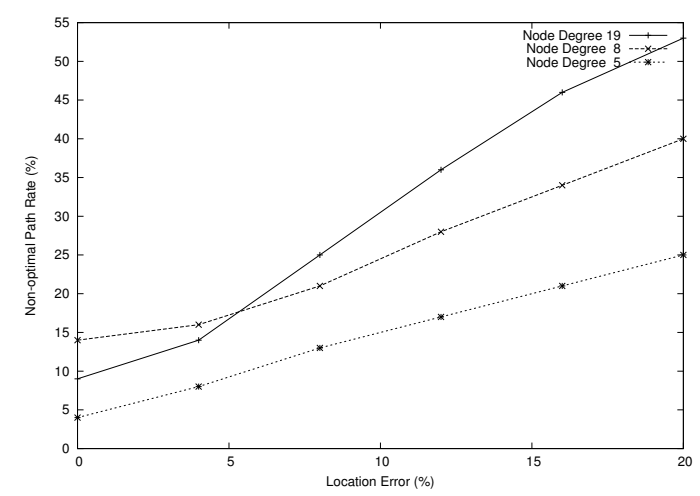

Figure 13: Impact of Location Inaccuracy and Node Degree on Non-optimal Path

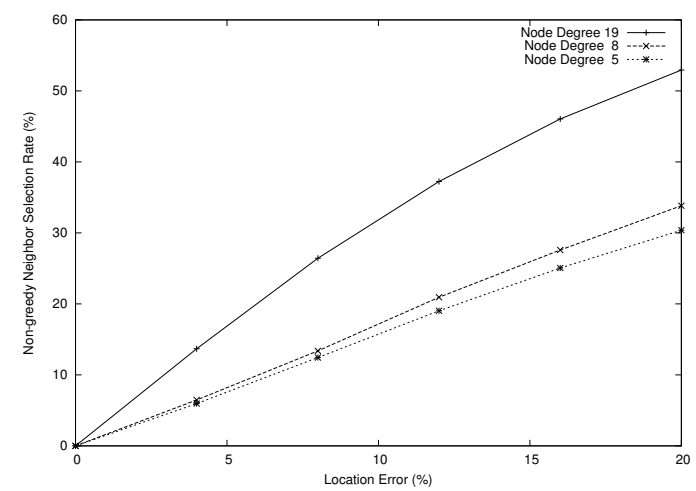

Figure 14: Wrong-greedy Neighbor Decision Rate

outweighed the good effects as can be clearly seen in the figure. This is because of stringent condition that should be satisfied for good impact to occur as was mentioned in section III.

\section{Analysis and Simulation of Perimeter Mode in GPSR:Case Study}

Local maximum resolution is one of the most important issues in geographic routing. Currently, graph-based solution such as face-2[14] or perimeter mode[1] using planar graph are considered the most advanced schemes. We investigate how planar graph construction is affected by location inaccuracy. As a case study, we selected Gabriel Graph (GG) in the well-known greedy perimeter stateless routing (GPSR)[1] protocol.

\section{VI.A. Correctness Analysis of Perimeter Mode in GPSR}

Perimeter mode routing resolves the local maximum problem by routing around the void using the righthand traversal rule. In order to enable such feature

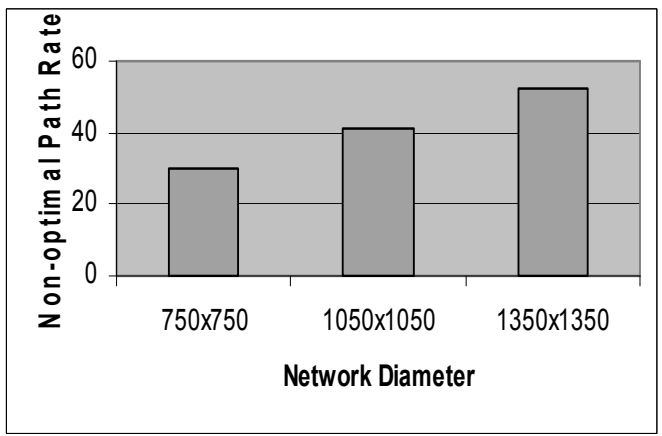

Figure 15: Impact of Location Inaccuracy and Network diameter on Non-optimal Path

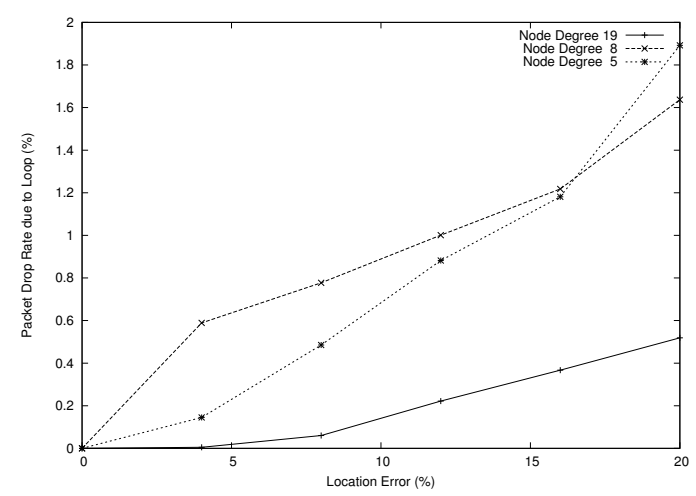

Figure 16: Packet Drop Rate Due to Routing Loop

all cross edges must be removed from the connectivity graph using planarization. One common way to establish planarization is through the Gabriel Graph (GG). In GG graph, there should be no witness within the circle between nodes to keep connection. As illustrated in figure 18, to keep connection between nodes $u$ and $v$, there should be no witness node $w$. Otherwise a cross edge is will be created, which prevents the packet from traversing the perimeter correctly. This condition is expressed as follows.

$$
\forall w \neq u, v: d^{2}(u, v)<\left[d^{2}(u, w)+d^{2}(v, w)\right]
$$

When there is inaccuracy in the location of $w$, such that the above condition is violated, then the planar graph of GG cannot be established correctly. In other words, if nodes $u$ and $v$ think that $w$ is outside the circle (due to absolute location inaccuracy) while in fact $w$ is indeed within the circle, then an edge that should be removed will not be removed. We call such scenario a planar graph collapse. If absolute location inaccuracy of node $w$ is $a$ and average distance between nodes is $2 r$ as described in figure 19, then the probability that planar graph collapses can be calculated as follows. 


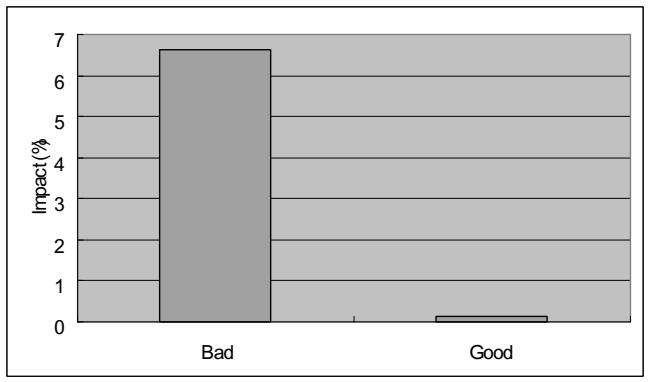

Figure 17: Good Imapct vs. Bad Impact of Location Inaccuracy

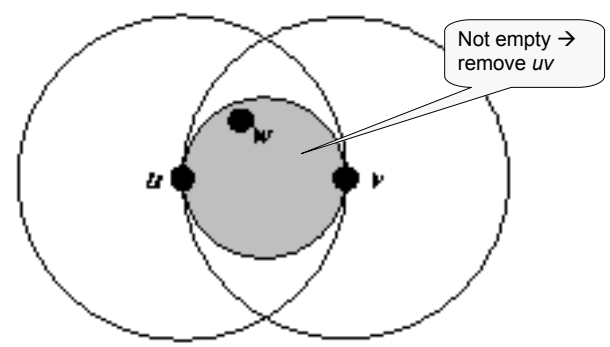

Figure 18: Planar Graph Construction

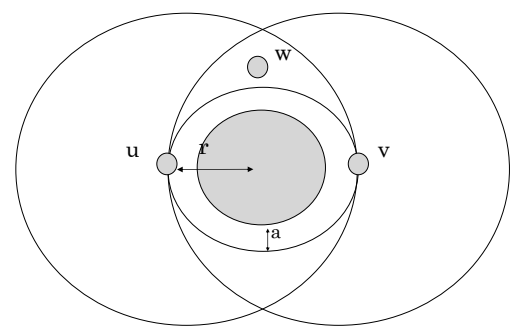

Figure 19: Correctness Analysis of Perimeter Mode of GPSR

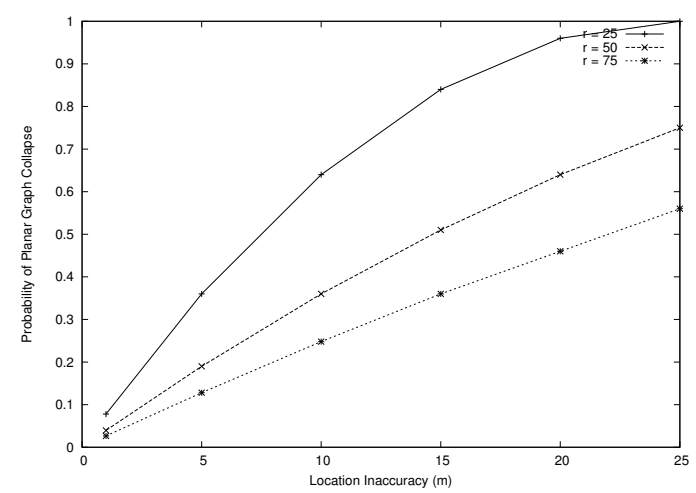

Figure 20: Probability of Planar Graph Collapse

perimeter mode in GPSR. Figure 21 shows planar graph error rate which represents the probability of planar graph collapse in our analysis. Simulation reProbability of Planar Graph Collapse $=\frac{\pi r^{2}-\pi(r-a)^{2}}{\pi r^{2}}$ sult trends match with our analysis result in that there is higher probability of planar graph collapse in denser network. Probability value is somewhat lower in simulation result because our analytical result is worst case as we mentioned in the previous section. We also note that not all planar graph collapse scenarios necessarily lead to packet drop. Furthermore, packet drop may be due to planar graph collapse or other effects of location inaccuracy (e.g., removal of edges that should not be removed). Figure 22 shows the packet drop rate due to location inaccuracy. As inaccuracy increase in perimeter mode, denser network show higher drop rate. However, in lower location error, sparser network shows higher drop rate, which is somewhat different from the probability of planar graph collapse. This occurs because there is higher probability of disconnection between nodes with even small location inaccuracy in sparser network. Figure 23 shows non-optimal path rate. Notice that nonoptimal rate is higher in sparse network even when
We perform simulation to compare with our earlier analysis and study impact of location inaccuracy on 


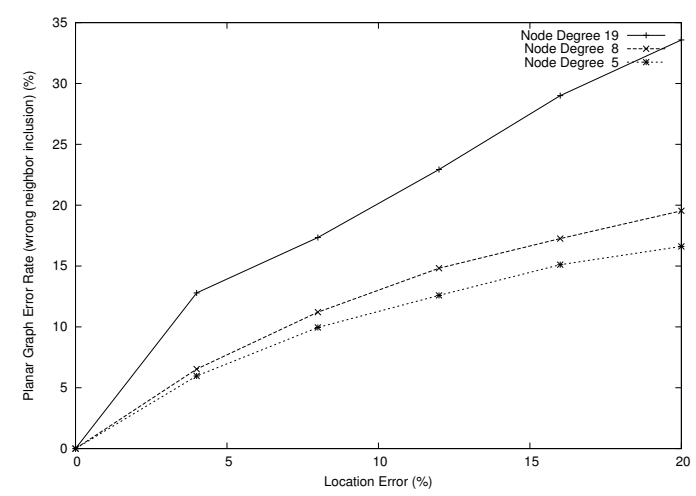

Figure 21: Planar Graph Error Rate

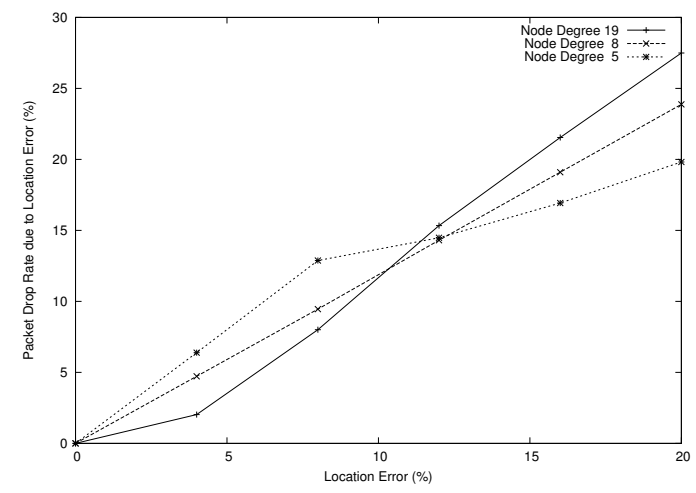

Figure 22: Packet Drop Rate Due to Location Inaccuracy in Perimeter Mode

location error is small. This is because there is higher probability of local maximum in sparse network resulting in detour path. In figure 24 , we can see that more routing loops happen in sparser network because of high relative distance inconsistency. One interesting result in this graph is that routing loop occurs in perimeter mode of GG graph even without location error. This is due to network disconnection and is not considered a protocol error per se.

\section{Summary and Conclusion}

In this paper, we have introduced a classification of location errors identifying four location inaccuracy metrics: (1) absolute location inaccuracy, (2) relative distance inaccuracy, (3) absolute location inconsistency and (4) relative distance inconsistency. Each of the location inaccuracy metrics affects geographic routing protocol and the impacts are summarized in table III. In the table, each inaccuracy metric is the cause of the problem and the impact.

Using extensive NS simulations, we conducted studies on greedy forwarding and perimeter mode routing. We observed the following impacts of location inaccuracy on geographic routing.

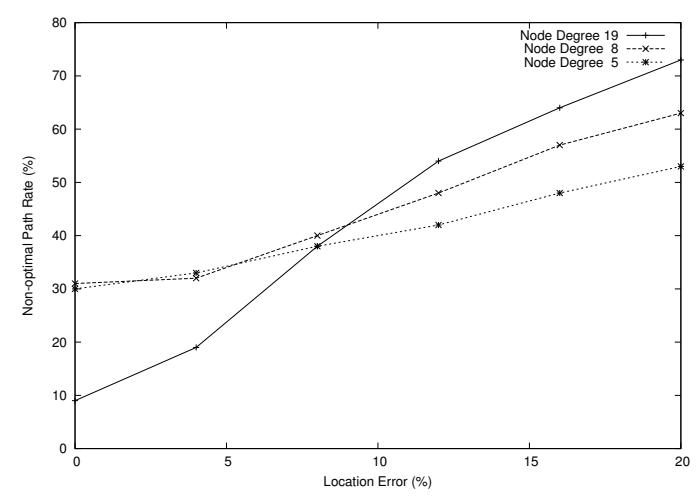

Figure 23: Non-optimal Path Rate in Perimeter Mode

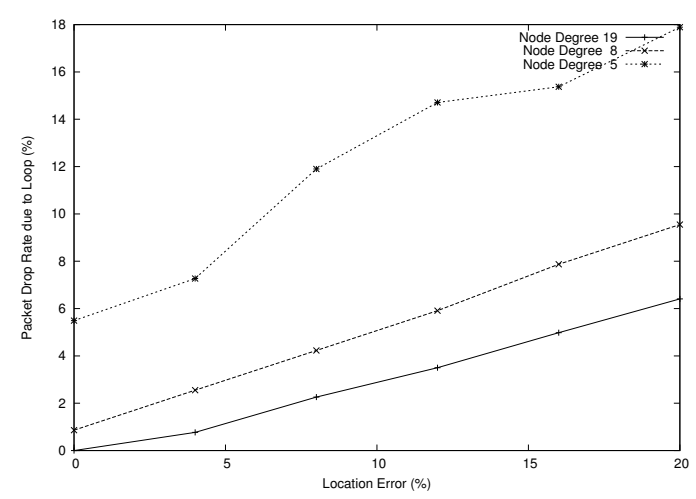

Figure 24: Impact on Loop in Perimeter Mode

(I) For greedy forwarding, packet drops occur mainly because of (a) routing loops caused by relative distance inconsistency and (b) no-route within destination caused by absolute location inconsistency. The packet drop rate is affected by the node degree and the network diameter. In denser network, there was more absolute location inconsistency due to shorter distance between neighbors which resulted in higher packet drop. In sparser network, there was more routing loops due to higher relative distance inconsistency, which resulted in packet drop. For the same network density, larger network diameter affects geographic routing more negatively in the presence of location in accuracy. This is because as the average path length increases, there is higher probability to incur relative distance inconsisency and absolute location inconsistency.

Non-optimal path is caused by relative distance inaccuracy. Relative distance inaccuracy causes wrong greedy neighbor selection. In addition, relative distance inaccuracy causes false local maximum which invokes local maximum resolution. Non-optimal path is affected by node degree and network diameter due to reasons similar to those of packet drops. Nonoptimal path is especially undesirable in wireless networks because it consumes valuable power. 
Table 3: Impact of Inaccuracy Metrics

\begin{tabular}{|c|c|c|c|}
\hline \multicolumn{2}{|c|}{ Inaccuracy Metrics } & Problem & Impact \\
\hline \hline \multirow{2}{*}{ Inaccuracy } & Absolute Location Inaccuracy & Wrong Neighbor Information & Packet Drop \\
\cline { 2 - 4 } & Relative Distance Inaccuracy & Wrong Greedy Decision & Non-optimal Path \\
\hline \multirow{2}{*}{ Inconsistency } & Absolute Location Inconsistency & False Local Maximum & Packet Drop \\
\cline { 2 - 4 } & Relative Distance Inconsistency & Wrong Greedy Decision & Routing Loop \\
\hline
\end{tabular}

(II) Location inaccuracy affects the correctness of graph-based local maximum resolution scheme. Our analysis of the perimeter mode (GG graph) of GPSR shows that in the presence of location inaccuracy, the planar graph is highly unlikely to be constructed correctly. Even with small absolute location inaccuracy, we observed high probability of planar graph collapse. Location inaccuracy degrades performance of perimeter mode in terms of packet drop, optimal path and routing loop rate.

The main contributions of this paper lie in (1) introducing classification and conditions of location related inaccuracies, (2) conducting the first in-depth analysis of the effects of location errors on geographic routing, and (3) uncovering severe performance degradation and even protocol correctness violations for geographic routing even in the presence of reasonable (relatively small) location errors, and even without considering mobility. This final remark is considered the major contribution of this paper, which points out that even with planarization and face-routing, geographic routing behaves incorrectly with location errors.

These observations indicate a pressing need to revisit the design of geographic routing protocols to be robust to location errors.

In the future we would like to investigate possible fixes to the problems uncovered in this paper. We hope that our work stimulates further research in this area, especially that several recent applications are being designed on top of geographic routing assuming their correctness[2,17].

\section{References}

[1] B.Karp and H.T.Kung., Greedy perimeter stateless routing for wireless networks, In Proc. of the 6th Annual ACM/IEEE Int. Conf. On Mobile Computing and Networking (MobiCom 2000), pages 243-254, Boston, MA, USA, 2000

[2] B. Greenstein, D. Estrin, R. Govindan, S. Ratnasamy, S. Shenker, DIFS: A Distributed Index for Features in Sensor Networks Proceedings of First IEEE International Workshop on Sensor Network Protocols and Applications Anchorage, AK. May 2003

[3] D. Son, J. Park, A. Helmy, Mobility-Induced Location Errors and its Effect on Geographic Routing in Ad Hoc Networks: Analysis and Improvement using Mobility Prediction, Poster, ACM MOBICOM Conference, September 2003

[4] E.Royer and C.K.Toh, A Review of Current Routing Protocols for Ad Hoc Wireless Networks, IEEE Pers. Commun., Apr., pp.46-55, 1999

[5] John Heidemann, Fabio Silva, Chalermek Intanagonwiwat, Ramesh Govindan, Deborah Estrin, and Deepak Ganesa, Building Efficient Wireless Sensor Networks with Low-Level Namin, In Proceedings of the Symposium on Operating Systems Principles, pp. 146-159. Chateau Lake Louise, Banff, Alberta, Canada, ACM. October, 2001

[6] J. Li, J. Jannotti, D. Couto, D. Karger, R. Morris, A Scalable Location Service for Geographic Ad Hoc Routing , ACM Mobicom 2000. The grid project homepage: http://www.pdos.lcs.mit.edu/grid

[7] K. Seada, A. Helmy, R. Govindan, On the Effect of Location Inaccuracy on Geographic Face Routing in Wireless Networks, Poster, ACM MOBICOM Conference, September 2003

[8] Karim Seada, A. Helmy, Ramesh Govindan, On the Effect of Localization Errors on Geographic Face Routing in Sensor Networks, Poster, The First ACM Conference on Embedded Networked Sensor Systems (ACM SenSys), November 2003

[9] K. Seada, A. Helmy, R. Govindan, On the Effect of Localization Errors on Geographic Face 
Routing in Sensor Networks, USC Technical Report, June 2003

[10] L.Blazevic, S.Giordano, and J.Le Boudec, Self Organized Terminode Routing, Tech. Rep. DSC/2000/040, Swiss Fed. Inst. Tech., Lausanne, Switzerland, 2000

[11] L. Breslau, D. Estrin, K. Fall, S. Floyd, J. Heidemann, A. Helmy, P. Huang, S. McCanne, K. Varadhan, Y. Xu, H. Yu, Advances in Network Simulation IEEE Computer, vol. 33, No. 5, p. 59-67, May 2000.

[12] M. Mauve, J. Sidmer, H. Hartenstein, A Survey on Position-Based Routing in Mobile AdHoc Networks, IEEE Network, Vol.15, pp.3039, 2001

[13] N. Bulusu, J. Heidemann, D. Estrin, T. Tran, Self-configuring Localization Systems: Design and Experimental Evaluation, ACM Transactions on Embedded Computing Systems (ACM TECS), Special issue on networked embedded systems, 2003

[14] P.Bose, P.Morin, I.Stojmenovic, and J.Urrutia, Routing and with guaranteed delivery in ad hoc wireless networks, In Proc. of 3rd ACM Intl. Workshop on Discrete Algorithms and Methods for Mobile Computing and Communications DIAL M99, pages 48-5, 1999

[15] P. Enge, P. Misra, Special Issue on Global Positioning System, Proceedings of the IEEE, Volume 87, No. 1, January, pp. 3-15 1999

[16] S.Basagni et al., A Distance Routing Effect Algorithm for Mobility(Dream), In Proc. 4th Annual ACM/IEEE Int. Conf. Mobile Computing and Networking, MOBICOM'98, Dalas, TX, USA, pp.76-84, 1998

[17] S. Ratnasamy, B. Karp, S. Shenker, D. Estrin, R. Govindan, L. Yin, F. Yu Data-Centric Storage in Sensornets with GHT, a Geographic Hash Table Mobile Networks and Applications (MONET), Journal of Special Issues on Mobility of Systems, Users, Data, and Computing: Special Issue on Algorithmic Solutions for Wireless, Mobile, Ad Hoc and Sensor Networks, Kluwer, mid-2003.

[18] S.Shenker, S.Ratnasamy, B.Karp, R.Govindan, D.Estrin, Data-centric storage in sensornets, Proceedings of the First ACM SIGCOMM
Workshop on Hot Topics in Networks(HotNets 2002), Princeton, NJ, October, 2002

[19] S.Slijepcevic, S.Megerian, M.Potkonjak, Location Errors in Wireless Embeded Sensor Networks:Sources, Models, and Effects on Applications, ACM Sigmobile Mobile Computing and Communication Review, vol.6,no.3,July,pp67782002

[20] Y.B.Ko and N.H.Vaidya., Location-Aided Routing (LAR) in mobile ad hoc networks. , ACM/Baltzer Wireless Networks(WINET) journal, 6(4):307-321, 2000

[21] Z.Haas and M.Pearlman, The Performance of Query Control Schemes for the Zone Routing Protocol, ACM/IEEE Trans. Net., Vol.9, No.4, Aug., pp.427-38, 2001

[22] Z.J.Haas and B.Liang, Ad-Hoc Mobility Management with Uniform Quorum Systems, IEEE/ACM Trans. Net., Vol.7, No.2, Apr., pp.228-40, 1999 